\title{
Detailed Nucleosynthesis Yields from the Explosion of Massive Stars
}

\author{
Carla Fröhlich $^{1}$, T. Fischer ${ }^{2}$, M. Liebendörfer ${ }^{2}$, F.-K. Thielemann ${ }^{2}$ \\ and J.W. Truran ${ }^{3,1}$ \\ ${ }^{1}$ Enrico Fermi Institute, University of Chicago, \\ 5640 South Ellis Avenue, Chicago IL 60637, USA \\ email: frohlich@uchicago.edu \\ ${ }^{2}$ Dept. of Physics, University of Basel, \\ Klingelbergstr. 82, CH-4056 Basel, Switzerland \\ ${ }^{3}$ Department of Astronomy and Astrophysics and ASC Flash Center, \\ 5640 S. Ellis Ave, Chicago, IL 60637, USA
}

\begin{abstract}
Despite the complexity and uncertainties of core collapse supernova simulations there is a need to provide correct nucleosynthesis abundances for the progressing field of galactic evolution and observations of low metallicity stars. Especially the innermost ejecta are directly affected by the explosion mechanism, i.e. most strongly the yields of Fe-group nuclei for which an induced piston or thermal bomb treatment will not provide the correct yields because the effect of neutrino interactions is not included.

Recent observations of metal-poor halo stars support the suggested existence of a lighter element primary process (LEPP) which operates very early in the galaxy and is independent of the r-process. We present a candidate for the LEPP, the so-called $\nu p$-process.
\end{abstract}

Keywords. nuclear reactions, nucleosynthesis, abundances - supernovae: general - neutrinos

\section{Introduction}

Massive stars end their life as core collapse supernovae. At the end of their hydrostatic evolution stars with main sequence masses $M \gtrsim 9 \mathrm{M}_{\odot}$ produce a core massive enough to undergo core collapse. This is the only fate for stars above $10 \mathrm{M}_{\odot}$ (see e.g. Heger et al. 2003), whereas in between stars can either collapse and form a neutron star or lose their envelope and become a white dwarf. The final stellar fate is determined by the size of the $\mathrm{CO}$ core. However, the relation of the size of the $\mathrm{CO}$ core to the progenitor mass depends on the metallicity. At higher metallicities mass loss becomes more important, producing smaller He and $\mathrm{CO}$ cores for a given initial mass. For very massive stars this can result in such a strong mass loss with increasing metallicity that black hole formation is excluded (leaving neutron stars as the only possible type of remnant). At low metallicities the final fate of a massive star depends on its initial mass: for initial masses of $\sim 10-25 \mathrm{M}_{\odot}$ as neutron star, for initial masses between $\sim 25 \mathrm{M}_{\odot}$ and $\sim 40 \mathrm{M}_{\odot}$ as black hole through fallback on the neutron star, or directly as black hole for initial masses above $\sim 40 \mathrm{M}_{\odot}$ (Hirschi et al. 2006, Nomoto et al. 2006, Blinnikov 2006). Core collapse with neutron star formation leads to supernovae (divided into subclasses, type II and type Ib/Ic, depending on the observation of $\mathrm{H}$ - and/or He-lines).

Such core collapse supernova events produce intermediate mass elements $\mathrm{Si}-\mathrm{Ca}$ and $\mathrm{Fe}$ and neighboring nuclei. The production of elements beyond Fe has long been postulated by three processes: the r-process, the s-process, and the p-process (or $\gamma$-process). The former two are caused by rapid or slow neutron captures. The latter stands for proton 
capture or alternative means to produce heavy neutron deficient, stable isotopes. The s-process takes place during stellar evolution and acts through neutron captures on $\mathrm{Fe}$ produced in previous stellar generations. The s-process is thus a "secondary" process. For the r-process and the p-process the location, operation, and uniqueness in astrophysical sites are still under debate. The r-process is required to be a primary process (Sneden and Cowan 2003); the production of such elements is independent of the initial heavy element content of the star. Most of the p-nuclei are thought to the produced in hot (supernova) environments through photodisintegration of preexisting heavy nuclei. This can account for the heavy p-nuclei but underproduces the light ones (see e.g. Arnould \& Goriely 2003, Costa et al. 2000, Hayakawa et al. 2004). The production mechanism for the light p-nuclei ${ }^{92,94} \mathrm{Mo}$ and ${ }^{96,98} \mathrm{Ru}$ is currently unknown. From chemical evolution studies of the cosmochronometer ${ }^{92} \mathrm{Nb}$ (Dauphas et al. 2003) a primary (supernova) origin is inferred.

The enrichment of the interstellar medium with these elements heavier than $\mathrm{H}, \mathrm{He}$, and $\mathrm{Li}$ (which originate from the Big Bang) can be traced via the surface composition of low mass stars of different ages. These stars are unaltered since their formation and therefore measure the composition in the interstellar medium at the time of their birth. Observations of such "metal-poor" stars provide information about the nucleosynthesis processes at the earliest times in the evolution of our Galaxy. The recently discovered hyper-metal-poor stars in the Milky Way may witness the chemical enrichment of the first generation of massive stars (the fastest evolving species).

\section{Core Collapse Supernova Nucleosynthesis}

Observations of supernova remnants shows typical kinetic energies of $10^{51} \mathrm{erg}$. Introducing a shock of appropriate energy in the pre-collapse stellar model (Woosley \& Weaver 1995, Thielemann et al. 1996, Nomoto et al. 1997, Hoffman et al. 1999, Nakamura et al. 1999, Rauscher et al. 2002, Nomoto et al. 2006) — either through a piston or through a thermal bomb - allows to perform nucleosynthesis calculations. However, such induced nucleosynthesis calculations are not self-consistent: they miss detailed knowledge of the explosion mechanism and of the location of the mass cut between the neutron star and the supernova ejecta. Therefore, they cannot predict the ejected ${ }^{56} \mathrm{Ni}$ masses from the innermost layers (undergoing explosive Si-burning) which powers the supernova light curves via the decay chain ${ }^{56} \mathrm{Ni}-{ }^{56} \mathrm{Co}-{ }^{56} \mathrm{Fe}$. The situation is different for the intermediate mass elements $\mathrm{Si}-\mathrm{Ca}$. They only depend on the explosion energy and the stellar structure of the progenitor star. Even lighter elements such as $\mathrm{O}$ and $\mathrm{Mg}$ are determined by the stellar evolution of the progenitor. Thus, when moving in from the outermost to the innermost ejecta of SN II explosion, we see an increase in the complexity, depending (a) only on stellar evolution, (b) on the stellar evolution and explosion energy, and (c) on stellar evolution and the complete explosion mechanism.

The correct prediction of the amount of Fe-group nuclei ejected (which includes also one of the so-called alpha-elements, i.e. $\mathrm{Ti}$ ) and their relative composition depends directly on the explosion mechanism and the size of the Fe core. Three types of uncertainties are inherent in the Fe-group ejecta, related to (i) the total amount of Fe(group) nuclei ejected and the mass cut between neutron star and ejecta, mostly measured by ${ }^{56} \mathrm{Ni}$ decaying to ${ }^{56} \mathrm{Fe}$, (ii) the total explosion energy which influences the entropy of the ejecta and with it the amount of radioactive ${ }^{44} \mathrm{Ti}$ as well as ${ }^{48} \mathrm{Cr}$ (decaying to ${ }^{48} \mathrm{Ti}$ and being responsible for elemental Ti), and (iii) finally the neutron richness or $Y_{e}=\langle Z / A>$ of the ejecta, dependent on stellar structure, electron captures, and neutrino interactions (Fröhlich et al. 2006a). The electron fraction $Y_{e}$ influences strongly the overall $\mathrm{Ni} / \mathrm{Fe}$ ratio. 
An example for the composition after explosive processing due to an (induced) shock wave is discussed in detail in Thielemann et al. 1996. The outer ejected layers $(M(r)>$ $\left.2 \mathrm{M}_{\odot}\right)$ are unprocessed by the explosion and contain results of prior $\mathrm{H}-, \mathrm{He}-, \mathrm{C}-$, and Ne-burning in stellar evolution. The interior parts of SNe II contain products of explosive Si, O, and Ne burning. In the inner ejecta, which experience explosive Si-burning, $Y_{e}$ changes from 0.4989 to 0.494 . The $Y_{e}$ originates from beta-decays and electron captures in the pre-explosive hydrostatic fuel in these layers. Neutrino reactions during the explosion were not yet included in these induced explosion calculations, utilizing a thermal bomb prescription. Huge changes occur in the Fe-group composition for mass zones below $M(r)=1.63 \mathrm{M}_{\odot}$. There the abundances of ${ }^{58} \mathrm{Ni}$ and ${ }^{56} \mathrm{Ni}$ become comparable. All neutron-rich isotopes $\left({ }^{57} \mathrm{Ni},{ }^{58} \mathrm{Ni},{ }^{59} \mathrm{Cu},{ }^{61} \mathrm{Zn},{ }^{62} \mathrm{Zn}\right)$ increase, the even-mass isotopes $\left({ }^{58} \mathrm{Ni},{ }^{62} \mathrm{Zn}\right)$ show the strongest effect. One can also recognize the increase of ${ }^{40} \mathrm{Ca},{ }^{44} \mathrm{Ti}$, ${ }^{48} \mathrm{Cr}$, and ${ }^{52} \mathrm{Fe}$ for the inner high entropy zones, but a reduction of the $N=Z \mathrm{nu}$ clei in the more neutron-rich layers. More details can be found in extended discussions (Thielemann et al. 1996, Nakamura et al. 1999).

\subsection{The $\nu$ p-process}

While the influence of neutrino interactions on supernova nucleosynthesis has been emphasized for many years only recently a new nucleosynthesis mechanism involving neutrinos has been identified to operate in core collapse supernovae. Recent core collapse supernova simulations with accurate neutrino transport (Liebendörfer et al. 2001, Buras et al. 2003, Thompson et al. 2005) show the presence of proton-rich neutrino heated matter, both in the inner ejecta (Liebendörfer et al. 2001, Buras et al. 2003) and the early neutrino wind from the proto-neutron star (Buras et al. 2003). This matter, part of the initially shock heated material located between the surface of the proto-neutron star and the shock front expanding through the outer layers, is subject to a large neutrino energy deposition heating the matter. This and the expansion, lifting the electron degeneracy, make it possible for the reactions $\nu_{e}+n \leftrightarrow p+e^{-}$and $p+\bar{\nu}_{e} \leftrightarrow n+e^{+}$(i.e. neutrino and antineutrino captures on free nucleons and their inverse reactions, electron and positron capture) to drive the composition proton-rich (Fröhlich et al. 2005, Pruet et al. 2005, Fröhlich et al. 2006a), i.e. the electron fraction $Y_{e}>0.5$. This effect will always be present in successful explosion with ejected matter irradiated by a strong neutrino flux, independent of the details of the explosion. While this matter expands and cools, nuclei can form. This results in a composition dominated by $N=Z$ nuclei, mainly ${ }^{56} \mathrm{Ni}$ and ${ }^{4} \mathrm{He}$, and protons. Without the further inclusion of neutrino and antineutrino reactions the composition of this matter will finally consist of protons, alpha-particles, and heavy (Fe-group) nuclei, i.e. a proton- and alpha-rich freeze-out that results in enhanced abundances of ${ }^{45} \mathrm{Sc},{ }^{49} \mathrm{Ti}$, and ${ }^{64} \mathrm{Zn}$ (Fröhlich et al. 2005, Pruet et al. 2005, Fröhlich et al. 2006a).

Traditional explosive (supernova) nucleosynthesis calculations did not include interactions with neutrinos and antineutrinos. The heaviest nuclei synthesized in these calculations have a mass number $A=64$. The matter flow stops at the nucleus ${ }^{64} \mathrm{Ge}$ which has a small proton capture probability and a beta-decay half-life (64s) that is much longer than the expansion time scale (10s) (Pruet et al. 2005). When reactions with neutrinos and antineutrinos are considered for both free and bound nucleons the situation becomes dramatically different (Fröhlich et al. 2006b, Pruet et al. 2006, Wanajo 2006).

$N \sim Z$ nuclei are practically inert to neutrino capture (converting a neutron into a proton) because such reactions are endoergic for neutron-deficient nuclei located away from the valley of stability. The situation is different for antineutrinos that are captured in a typical time of a few seconds, both on protons and on nuclei, at the distances at which 
nuclei form $(\sim 1000 \mathrm{~km})$. As protons are more abundant than heavy nuclei, antineutrino capture occurs predominantly on protons, causing a residual density of free neutrons of $10^{14}-10^{15} \mathrm{~cm}^{-3}$ for several seconds when the temperatures are in the range $1-3 \times 10^{9} \mathrm{~K}$. This effect is clearly seen in Figure 1 of Fröhlich et al. 2006a where the time evolution of the abundances of protons, neutrons, alpha-particles, and ${ }^{56} \mathrm{Ni}$ is shown for a trajectory of the model B07. The solid (dashed) lines display the nucleosynthesis results which include (omit) neutrino and antineutrino absorption interactions after nuclei are formed. ${ }^{56} \mathrm{Ni}$ serves to illustrate when nuclei are formed. The difference in proton abundances between both calculations is due to antineutrino captures on protons, producing neutrons which drive the $\nu p$-process. Without the inclusion of antineutrino captures the neutron abundance soon becomes too small to allow for any capture on heavy nuclei.

The neutrons produced via antineutrino absorption on protons can easily be captured by neutron-deficient $N \sim Z$ nuclei (for example ${ }^{64} \mathrm{Ge}$ ) which have large neutron capture cross sections. While proton capture, $(\mathrm{p}, \gamma)$, on ${ }^{64} \mathrm{Ge}$ takes too long or is impossible, the $(\mathrm{n}, \mathrm{p})$ reaction dominates, permitting the matter flow to continue to nuclei heavier than ${ }^{64} \mathrm{Ge}$ via subsequent proton captures with freeze-out close to $1 \times 10^{9} \mathrm{~K}$.

Figure 2 of Fröhlich et al. 2006a shows the results for the composition of supernova ejecta from one hydrodynamical model which includes neutrino absorption reactions in the nucleosynthesis calculations (filled circles) that lead initially to proton-rich conditions in the innermost zones, experiencing afterwards the $\nu p$-process. These abundances are compared to an older set of nucleosynthesis calculations (open circles, Thielemann et al. 1996) that did not include neutrino interactions and therefore did not produce the protonrich matter resulting in models with accurate neutrino transport (Liebendörfer et al. 2001, Buras et al. 2003, Thompson et al. 2005). In later phases of the cooling protoneutron star neutrino interactions will cause neutron-rich ejecta. Whether this permits weak or strong r-process is still debated (Thompson 2003).

\section{Evidence for a Lighter Element Primary Process (LEPP)}

Metal-poor stars in the Galactic halo provide us with a laboratory to study the earliest Galactic nucleosynthesis processes (Cowan \& Sneden 2007 and references therein). Their chemical composition also provides us with hints about the nature of the very first generation of stars. Early observations (e.g. Spite and Spite 1978, Gilroy et al. 1988, Gratton \& Sneden 1994, Burris et al. 2000) focussed on stars with metallicites of $[\mathrm{Fe} / \mathrm{H}]<-1$ and rare earth elements which are easily detectable with ground-based telescopes. However, with the latest generation of telescopes and with space based telescopes, such as the Hubble Space Telescope (HST), a much larger range of n-capture elements can be examined. The most metal-poor stars observed to date, HE0107-5240 (Christlieb et al. 2002) and HE1327-2326 (Frebel et al. 2005), with metallicities below $[\mathrm{Fe} / \mathrm{H}]<-5$ are enriched in $\mathrm{C}, \mathrm{N}$, and $\mathrm{O}$ but very poor in n-capture elements. However, the detection of $\mathrm{Sr} / \mathrm{Fe}$ (exceeding 10 times the solar ratio) in the most metal-poor star (HE1327-2326, Frebel et al. 2005) suggests the existence of a primary process producing elements beyond $\mathrm{Fe}$ and $\mathrm{Zn}$.

Based on abundance data from HST observations of metal-poor galactic halo stars (Cowan et al. 2005) analyze the behavior of Ge versus metallicity $[\mathrm{Fe} / \mathrm{H}]$. The observed trend (see Figs. 4 and 5 of Cowan et al. 2005) is consistent with an explosive (or charged particle) synthesis for Ge. A similar comparison for $\mathrm{Zr}$ is somewhat less conclusive but nevertheless seems to indicate a different synthesis origin for these two elements.

François et al. 2007 obtained abundances for 16 n-capture elements from a sample of 32 extremely metal-poor stars. Their measurements imply that not all n-capture elements 
in metal-poor stars were produced by a single r-process. From this they conclude that an additional process must contribute mainly to the production of the first peak elements in very metal-poor stars and extremely metal-poor stars.

Recent galactic chemical evolution studies (Travaglio et al. 2004a) of the Galactic enrichment of Sr, Y, and $\mathrm{Zr}$ (using homogeneous chemical models) suggest the existence of additional primary process, denoted lighter element primary process (LEPP), to explain the observed abundances. This process is independent of the r-process and operates very early in the Galaxy. Travaglio et al. 2004a point to massive stars as likely site for this process. The mean residuals of Sr, Y, and Zr in the François et al. 2007 sample (based on the Solar-system r-process abundances by Arlandini et al. 1999) show that this LEPP process is responsible for $90-95 \%$ of the total abundance of these elements at $[\mathrm{Ba} / \mathrm{H}] \simeq-4.3$. In a recent paper by Montes et al. 2007 the authors derive an abundance pattern of the LEPP and explore the possibility of a neutron-capture process.

A candidate for the LEPP is the $\nu p$-process (Fröhlich et al. 2006b). This nucleosynthesis process will occur in all core collapse supernovae and could explain the existence of $\mathrm{Sr}$ and other elements beyond Fe in the very early stage of galactic evolution. This process can also contribute to the nucleosynthesis of light p-process elements.

\section{References}

Arlandini, C, Käppeler, F., Wishak, K., et al. 1999 ApJ, 525, 886

Arnould, M. \& Goriely, S. 2003, Phys. Rep., 384, 1

Blinnikov, S. 2006, Surv. High Energy Phys., 20, 89

Buras, R., Rampp, M., Janka, H.-T., et al. 2003, Phys. Rev. Letters, 90, 24, 241101

Burris D. L., Pilachowski, C. A., Armandroff, T. E. et al. 2000, ApJ, 544, 302

Cayrel, R., Depagne, E., Spite, M. et al. 2004 A\&A, 416, 1117

Christlieb, N., Bessell, M. S., Beers, T. C. et al. 2002, Nature, 419, 904

Costa, V. Rayet, M. Zappala, R. A. et al. 2000, A\&A, 358, L67

Cowan, J. J., \& Sneden, C. 2007, Nature, 440, 1151

Cowan, J. J., Sneden, C., Beers, T. C. et al. 2005, ApJ, 627, 238

Dauphas, N., Rauscher, T., Marty, B. et al. 2003, Nucl. Phys. A, 719, C287

Domínguez, I., Höflich, P., and Straniero, O. 2001, ApJ, 557, 279

François, P., Depagne, E., Hill, V. et al. 2007, A\&SA, 476, 935

François, P., Matteucci, F. \& Cayrel, R. et al. 2004, A\&A, 421, 613

Frebel, A., Aoki, W., Christlieb, N., et al. 2005, Nature, 434, 871

Fröhlich, C., Hauser, P., Liebendörfer, M. et al. 2005, Nucl. Phys. A, 758, 27

Fröhlich, C., Hauser, P., Liebendörfer, M. et al. 2006, ApJ, 637, 415

Fröhlich, C., Martínez-Pinedo, G., Liebendörfer, M. et al. 2006, Phys. Rev. Letters, 96, 14, 142502

Gilroy, K. K., Sneden, C., Pilachowski, C. A., \& Cowan, J. J. 1988, ApJ, 327, 298

Gratton, R. G. \& Sneden, C. 1994, A\& $A, 287,927$

Gratton, R. G. \& Sneden, C. 1991, A\& $A, 241,501$

Hayakawa, T., Iwamoto, N., Shizuma, T. et al. 2004, Phys. Rev. Lett., 93, 16, 161102

Heger, A., Fryer, C. L., Woosley, S. E. et al. 2003, ApJ, 591, 288

Hirschi, R., Meynet, G., and Maeder, A. 2006,

Hoffman, R. D., Woosley, S. E., Weaver et al. 1999, ApJ, 521, 735

Khokhlov, A. M., Höflich, P. A., Oran, E. S. et al. 1999, ApJ Lett., 524, L107

Langanke, K. \& Martínez-Pinedo, G. 2000, Nucl. Phys. A, 673, 481

Liebendörfer, M., Mezzacappa, A., \& Thielemann et al. 2001, Phys. Review D, 6310, 103004

Liebendörfer, M., Mezzacappa, A., Messer, O. E. B. et al. 2003, Nucl. Phys. A, 719, 144

Livio, M., Panagia, N., \& Sahu, K. (eds.), 2001, Supernovae and gamma-ray bursts: the greatest explosions since the Big Bang (Cambridge: CUP)

Lodders, K. 2003, ApJ, 591, 1220 
Montes, F. Beers, T. C., Cowan, J. et al. 2007, ApJ, 671, 1685

Nakamura, T., Umeda, H., Iwamoto, K. et al. 2001, ApJ, 555, 880

Nakamura, T., Umeda, H., Nomoto, K. et al. 1999, ApJ, 517, 193

Nomoto, K., Tominaga, N., Umeda, H. et al. 2006, Nucl. Phys. A, 777, 424

Nomoto, K., Hashimoto, M., Tsujimoto, T. et al. 1997, Nucl. Phys. A, 616, 79

Pruet, J., Hoffman, R. D., Woosley, S. E. et al. 2006, ApJ, 644, 1028

Pruet, J., Woosley, S. E., Buras, R. et al. 2005, ApJ, 623, 325

Rauscher, T., Heger, A., Hoffman, R. D. et al. 2002, ApJ, 576, 323

Sneden, C. \& Cowan, J. J. 2003, Science, 299, 70

Spite, M. \& Spite, F. 1978, A\&A A, 67, 23

Thielemann, F.-K., Nomoto, K., \& Hashimoto, M. 1996, ApJ, 460, 408

Thompson, T. A. 2003, ApJ, 585, L33

Thompson, T. A., Quataert, E., \& Burrows, A. 2005, ApJ, 620, 861

Timmes, F. X., Woosley, S. E., \& Weaver, T. A. 1995, ApJ Suppl., 98, 617

Travaglio, C., Gallino, R., Arnone, E. et al. 2004, ApJ, 601, 864

Travaglio, C., Hillebrandt, W., Reinecke, M. et al. 2004, A\&SA, 425, 1029

Wanajo, S. 2006, ApJ, 647, 1323

Woosley, S. E. 1993, ApJ, 405, 273

Woosley, S. E. \& Weaver, T. A. 1994, in: Bludman, S. A., Mochkovitch, R., \& Zinn-Justin, J. (eds.), Supernovae, (Amsterdam: Elsevier Science), NATO Advanced Sci Inst Ser C, 63

Woosley, S. E. \& Weaver, T. A. 1995, ApJ Suppl., 101, 181

\section{Discussion}

LIMONGI: Which is the main reason for the increase of $[\mathrm{Sc} / \mathrm{Fe}]$ and $[\mathrm{Cu} / \mathrm{Fe}]$ in your models?

FröHlich: The $[\mathrm{Sr} / \mathrm{Fe}]$ and $[\mathrm{Cu} / \mathrm{Fe}]$ ratios in our models are increased compared to earlier calculations, e.g. Thielemann et al. (1996), due to the proton-rich material which emerges from the core collapse simulation. Even if we switch off the neutrino interactions in the proton-rich ejecta we still get these increased ratios for $\mathrm{Sc}$ and $\mathrm{Cu}$ which are consistent with observations of metal-poor stars.

MAEDER: The elements Sr, Y, Zr are typical s-elements which can be created by the s-process during the He-burning phase of low $\mathrm{Z}$ massive stars and not necessarily in the supernova explosions. In my opinion, these excesses of $\mathrm{Sr}, \mathrm{Y}, \mathrm{Zr}$ are related to the $\mathrm{N}$-excesses (as well as $\mathrm{C}, \mathrm{O}$ ) prominently observed in this extreme star (Frebel star).

FRÖHLICH: Recent observations of extremely metal-poor stars e.g. by François et al. show clear indications for the need of an additional primary process (independent of the r-process) to explain the observed abundance pattern in these stars. The question is down to which metallicity the s-process, being a secondary process, can still operate. 